\title{
SPHERICAL CLASSES AND THE ALGEBRAIC TRANSFER
}

\author{
NGUYỄNN H. V. HU'NG
}

\begin{abstract}
We study a weak form of the classical conjecture which predicts that there are no spherical classes in $Q_{0} S^{0}$ except the elements of Hopf invariant one and those of Kervaire invariant one. The weak conjecture is obtained by restricting the Hurewicz homomorphism to the homotopy classes which are detected by the algebraic transfer.

Let $P_{k}=\mathbb{F}_{2}\left[x_{1}, \ldots, x_{k}\right]$ with $\left|x_{i}\right|=1$. The general linear group $G L_{k}=$ $G L\left(k, \mathbb{F}_{2}\right)$ and the $(\bmod 2)$ Steenrod algebra $\mathcal{A}$ act on $P_{k}$ in the usual manner. We prove that the weak conjecture is equivalent to the following one: The canonical homomorphism $j_{k}: \mathbb{F}_{2} \otimes\left(P_{k}^{G L_{k}}\right) \rightarrow\left(\mathbb{F}_{2} \otimes P_{k}\right)^{G L_{k}}$ induced by the identity map on $P_{k}$ is zero in positive dimensions for $k>2$. In other words, every Dickson invariant (i.e. element of $P_{k}^{G L_{k}}$ ) of positive dimension belongs to $\mathcal{A}^{+} \cdot P_{k}$ for $k>2$, where $\mathcal{A}^{+}$denotes the augmentation ideal of $\mathcal{A}$. This conjecture is proved for $k=3$ in two different ways. One of these two ways is to study the squaring operation $S q^{0}$ on $P\left(\mathbb{F}_{2} \otimes P_{k}^{*}\right)$, the range of $j_{k}^{*}$, and $G L_{k}$

to show it commuting through $j_{k}^{*}$ with Kameko's $S q^{0}$ on $\mathbb{F}_{2} \otimes P\left(P_{k}^{*}\right)$, the $G L_{k}$

domain of $j_{k}^{*}$. We compute explicitly the action of $S q^{0}$ on $P\left(\mathbb{F}_{2} \otimes P_{k}^{*}\right)$ for $k \leq 4$.
\end{abstract}

\section{INTRODUCTION}

The paper deals with the spherical classes in $Q_{0} S^{0}$, i.e. the elements belonging to the image of the Hurewicz homomorphism

$$
H: \pi_{*}^{s}\left(S^{0}\right) \cong \pi_{*}\left(Q_{0} S^{0}\right) \rightarrow H_{*}\left(Q_{0} S^{0}\right) .
$$

Here and throughout the paper, the coefficient ring for homology and cohomology is always $\mathbb{F}_{2}$, the field of 2 elements.

We are interested in the following classical conjecture.

Conjecture 1.1. (conjecture on spherical classes). There are no spherical classes in $Q_{0} S^{0}$, except the elements of Hopf invariant one and those of Kervaire invariant one.

(See Curtis [9] and Wellington [21] for a discussion.)

Let $V_{k}$ be an elementary abelian 2-group of rank $k$. It is also viewed as a $k$ dimensional vector space over $\mathbb{F}_{2}$. So, the general linear group $G L_{k}=G L\left(k, \mathbb{F}_{2}\right)$

Received by the editors April 7, 1995.

1991 Mathematics Subject Classification. Primary 55P47, 55Q45, 55S10, 55T15.

Key words and phrases. Spherical classes, loop spaces, Adams spectral sequences, Steenrod algebra, invariant theory, Dickson algebra, algebraic transfer.

The research was supported in part by the DGU through the CRM (Barcelona).

(C)1997 American Mathematical Society 
acts on $V_{k}$ and therefore on $H^{*}\left(B V_{k}\right)$ in the usual way. Let $D_{k}$ be the Dickson algebra of $k$ variables, i.e. the algebra of invariants

$$
D_{k}:=H^{*}\left(B V_{k}\right)^{G L_{k}} \cong \mathbb{F}_{2}\left[x_{1}, \ldots, x_{k}\right]^{G L_{k}},
$$

where $P_{k}=\mathbb{F}_{2}\left[x_{1}, \ldots, x_{k}\right]$ is the polynomial algebra on $k$ generators $x_{1}, \ldots, x_{k}$, each of dimension 1 . As the action of the $(\bmod 2)$ Steenrod algebra $\mathcal{A}$ and that of $G L_{k}$ on $P_{k}$ commute with each other, $D_{k}$ is an algebra over $\mathcal{A}$.

One way to attack Conjecture 1.1 is to study the Lannes-Zarati homomorphism

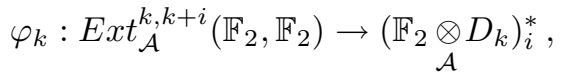

which is compatible with the Hurewicz homomorphism (see [12], [13, p.46]). The domain of $\varphi_{k}$ is the $E_{2}$-term of the Adams spectral sequence converging to $\pi_{*}^{s}\left(S^{0}\right) \cong$ $\pi_{*}\left(Q_{0} S^{0}\right)$. Furthermore, according to Madsen's theorem [15] which asserts that $D_{k}$ is dual to the coalgebra of Dyer-Lashof operations of length $k$, the range of $\varphi_{k}$ is a submodule of $H_{*}\left(Q_{0} S^{0}\right)$. By compatibility of $\varphi_{k}$ and the Hurewicz homomorphism we mean $\varphi_{k}$ is a "lifting" of the latter from the " $E_{\infty}$-level" to the " $E_{2}$-level".

Let $h_{r}$ denote the Adams element in $\operatorname{Ext}_{\mathcal{A}}^{1,2^{r}}\left(\mathbb{F}_{2}, \mathbb{F}_{2}\right)$. Lannes and Zarati proved in [13] that $\varphi_{1}$ is an isomorphism with $\left\{\varphi_{1}\left(h_{r}\right) \mid r \geq 0\right\}$ forming a basis of the dual of $\mathbb{F}_{2} \otimes D_{1}$ and $\varphi_{2}$ is surjective with $\left\{\varphi_{2}\left(h_{r}^{2}\right) \mid r \geq 0\right\}$ forming a basis of the dual of $\mathbb{F}_{2} \otimes \stackrel{\mathcal{A}}{D_{2}}$. Recall that, from Adams [1], the only elements of Hopf invariant one are

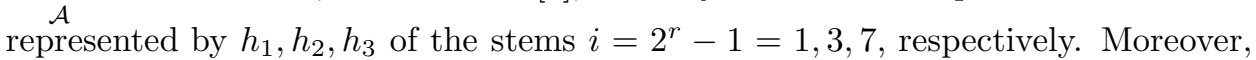
by Browder [5], the only dimensions where an element of Kervaire invariant one would occur are 2(2r -1$)$, for $r>0$, and it really occurs at this dimension if and only if $h_{r}^{2}$ is a permanent cycle in the Adams spectral sequence for the spheres.

Therefore, Conjecture 1.1 is a consequence of the following:

Conjecture 1.2. $\varphi_{k}=0$ in any positive stem $i$ for $k>2$.

It is well known that the Ext group has intensively been studied, but remains very mysterious. In order to avoid the shortage of our knowledge of the Ext group, we want to restrict $\varphi_{k}$ to a certain subgroup of Ext which (1) is large enough and worthwhile to pursue and (2) could be handled more easily than the Ext itself. To this end, we combine the above data with Singer's algebraic transfer.

Singer defined in [20] the algebraic transfer

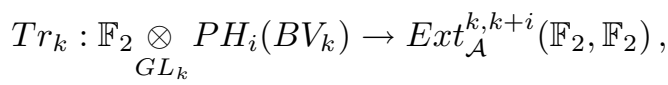

where $P H_{*}\left(B V_{k}\right)$ denotes the submodule consisting of all $\mathcal{A}$-annihilated elements in $H_{*}\left(B V_{k}\right)$. It is shown to be an isomorphism for $k \leq 2$ by Singer [20] and for $k=3$ by Boardman [4]. Singer also proved that it is an isomorphism for $k=4$ in a range of internal degrees. But he showed it is not an isomorphism for $k=5$. However, he conjectures that $T r_{k}$ is a monomorphism for any $k$.

Our main idea is to study the restriction of $\varphi_{k}$ to the image of $\operatorname{Tr}_{k}$.

Conjecture 1.3. (weak conjecture on spherical classes).

$$
\varphi_{k} \cdot T r_{k}: \mathbb{F}_{2} \underset{G L_{k}}{\otimes} P H_{*}\left(B V_{k}\right) \rightarrow P\left(\mathbb{F}_{2} \underset{G L_{k}}{\otimes} H_{*}\left(B V_{k}\right)\right):=\left(\mathbb{F}_{2} \underset{\mathcal{A}}{\otimes} D_{k}\right)^{*}
$$

is zero in positive dimensions for $k>2$. 
In other words, there are no spherical classes in $Q_{0} S^{0}$, except the elements of Hopf invariant one and those of Kervaire invariant one, which can be detected by the algebraic transfer.

A natural question is: How can one express $\varphi_{k} \cdot T r_{k}$ in the framework of invariant theory alone, and without using the mysterious Ext group?

Let $j_{k}: \mathbb{F}_{2} \otimes\left(P_{k}^{G L_{k}}\right) \rightarrow\left(\mathbb{F}_{2} \otimes P_{k}\right)^{G L_{k}}$ be the natural homomorphism induced by the identity map on $P_{k}$. We have

Theorem 2.1. $\varphi_{k} \cdot \operatorname{Tr}_{k}$ is dual to $j_{k}$, or equivalently,

$$
j_{k}=T r_{k}^{*} \cdot \varphi_{k}^{*}
$$

By this theorem, Conjecture 1.3 is equivalent to

Conjecture 1.4. $j_{k}=0$ in positive dimensions for $k>2$.

This seems to be a surprise, because by an elementary argument involving taking averages, one can see that if $H \subset G L_{k}$ is a subgroup of odd order then the similar homomorphism

$$
j_{H}: \mathbb{F}_{2} \otimes \underset{\mathcal{A}}{\otimes}\left(P_{k}^{H}\right) \rightarrow\left(\mathbb{F}_{2} \underset{\mathcal{A}}{\otimes P_{k}}\right)^{H}
$$

is an isomorphism. Furthermore, $j_{1}$ is iso and $j_{2}$ is mono.

Obviously, $j_{k}=0$ if and only if the composite

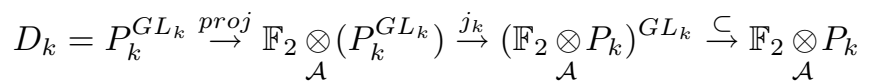

is zero. So, Conjecture 1.4 can equivalently be stated in the following form.

Conjecture 1.5. Let $D_{k}^{+}, \mathcal{A}^{+}$denote the augmentation ideals in $D_{k}$ and $\mathcal{A}$, respectively. Then $D_{k}^{+} \subset \mathcal{A}^{+} \cdot P_{k}$ for any $k>2$.

The domain and range of $j_{k}$ both are still mysterious. Anyhow, they seem easier to handle than the Ext group. They both are well-known for $k=1,2$. Furthermore, on the one hand, $\left(\mathbb{F}_{2} \underset{\mathcal{A}}{\otimes P_{k}}\right)^{G L_{k}}$ is computed for $k=3$ by Kameko [11], Alghamdi-Crabb-Hubbuck [3] and Boardman [4]. On the other hand, $\mathbb{F}_{2} \underset{\mathcal{A}}{\otimes}\left(P_{k}^{G L_{k}}\right)$ is determined by Hu'ng-Peterson [18] for $k=3$ and 4.

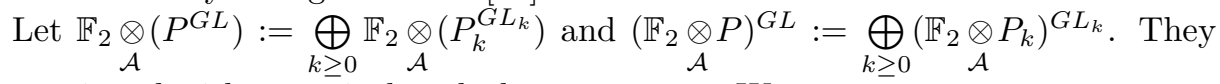
are equipped with canonical coalgebra structures. We get

Proposition 3.1. $\left.j=\bigoplus \underset{j_{k}}{ }: \mathbb{F}_{2} \underset{\mathcal{A}}{\otimes}\left(P^{G L}\right) \rightarrow \underset{\mathcal{A}}{\left(\mathbb{F}_{2} \otimes P\right.}\right)^{G L}$ is a homomorphism of coalgebras.

Let $S q^{0}: P H_{*}\left(B V_{k}\right) \rightarrow P H_{*}\left(B V_{k}\right)$ be Kameko's squaring operation that commutes with the Steenrod operation $S q^{0}: \operatorname{Ext}_{\mathcal{A}}^{k, t}\left(\mathbb{F}_{2}, \mathbb{F}_{2}\right) \rightarrow E x t_{\mathcal{A}}^{k, 2 t}\left(\mathbb{F}_{2}, \mathbb{F}_{2}\right)$ through the algebraic transfer $\operatorname{Tr}_{k}$ (see [11], [3], [4], [17]). Note that $S q^{0}$ is completely different from the identity map. We prove

Proposition 4.2. There exists a homomorphism

$$
S q^{0}: P\left(\mathbb{F}_{2} \underset{G L_{k}}{\otimes} H_{*}\left(B V_{k}\right)\right) \rightarrow P\left(\mathbb{F}_{2} \underset{G L_{k}}{\otimes} H_{*}\left(B V_{k}\right)\right)
$$

which commutes with Kameko's $S q^{0}$ through the homomorphism $j_{k}^{*}$.

These two propositions lead us to two different proofs of the following theorem. 
Theorem 3.2. $j_{k}^{*}=0$ in positive dimensions for $k=3$. In other words, there is no spherical class in $Q_{0} S^{0}$ which is detected by the triple algebraic transfer.

We compute explicitly the action of $S q^{0}$ on $P\left(\mathbb{F}_{2} \otimes H_{*}\left(B V_{k}\right)\right)$ for $k=3$ and 4 in Propositions 5.2 and 5.4. $G L_{k}$

The paper contains six sections and is organized as follows.

Section 2 is to prove Theorem 2.1. In Section 3, we assemble the $j_{k}$ for $k \geq 0$ to get a homomorphism of coalgebras $j=\bigoplus j_{k}$. By means of this property of $j$ we give there a proof of Theorem 3.2. Section 4 deals with the existence of the squaring operation $S q^{0}$ on $P\left(\mathbb{F}_{2} \otimes H_{*}\left(B V_{k}\right)\right)$ that leads us to an alternative proof for Theorem 3.2. This proof helps to explain the problem. In Section 5 , we compute explicitly the action of $S q^{0}$ on $P\left(\mathbb{F}_{2} \otimes H_{*}\left(B V_{k}\right)\right)$ for $k \leq 4$. Finally, in Section 6 we state a conjecture on the Dickson algebra that concerns spherical classes.

\section{ACKNOWLEDGMENTS}

I express my warmest thanks to Manuel Castellet and all my colleagues at the CRM (Barcelona) for their hospitality and for providing me with a wonderful working atmosphere and conditions. I am grateful to Jean Lannes and Frank Peterson for helpful discussions on the subject. Especially, I am indebted to Frank Peterson for his constant encouragement and for carefully reading my entire manuscript, making several comments that have led to many improvements.

\section{EXPRESSING $\varphi_{k} \cdot T r_{k}$ IN THE FRAMEWORK OF INVARIANT THEORY}

First, let us recall how to define the homomorphism $j_{k}$.

We have the commutative diagram

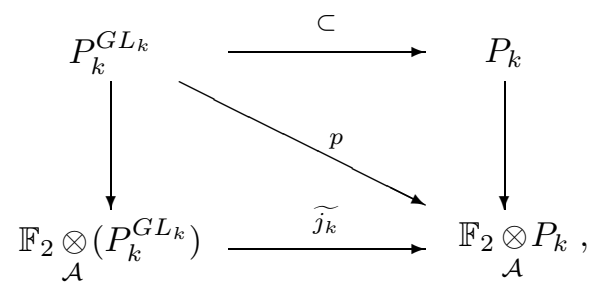

where the vertical arrows are the canonical projections, and $\widetilde{j_{k}}$ is induced by the inclusion $P_{k}^{G L_{k}} \subset P_{k}$. Obviously, $p\left(P_{k}^{G L_{k}}\right) \subset\left(\mathbb{F}_{2} \otimes P_{k}\right)^{G L_{k}}$. So, $\widetilde{j_{k}}$ factors through $\left(\mathbb{F}_{2} \otimes P_{\mathcal{A}}\right)^{G L_{k}}$ to give rise to

$$
\begin{aligned}
& j_{k}: \mathbb{F}_{2} \otimes\left(P_{k}^{G L_{k}}\right) \rightarrow\left(\mathbb{F}_{2} \otimes P_{k}\right)^{G L_{k}}, \\
& \underset{\mathcal{A}}{j_{k}^{\mathcal{A}}(1 \otimes Y)}=\quad \stackrel{\mathcal{A}}{1 \otimes Y} \underset{\mathcal{A}}{ },
\end{aligned}
$$

for any polynomial $Y \in D_{k}=P_{k}^{G L_{k}}$.

The goal of this section is to prove the following theorem.

Theorem 2.1. $j_{k}=\operatorname{Tr}_{k}^{*} \cdot \varphi_{k}^{*}$.

Now we prepare some data in order to prove the theorem at the end of this section. 
First we sketch Lannes-Zarati's work [13] on the derived functors of the destabilization. Let $\mathcal{D}$ be the destabilization functor, which sends an $\mathcal{A}$-module $M$ to the unstable $\mathcal{A}$-module $\mathcal{D}(M)=M / B(M)$, where $B(M)$ is the submodule of $M$ generated by all $S q^{i} u$ with $u \in M, i>|u|$.

$\mathcal{D}$ is a right exact functor. Let $\mathcal{D}_{k}$ be its $k$-th derived functor for $k \geq 0$.

Suppose $M_{1}, M_{2}$ are $\mathcal{A}$-modules. Lannes and Zarati defined in $[13, \S 2]$ a homomorphism

$$
\begin{aligned}
\cap: \operatorname{Ext}_{\mathcal{A}}^{r}\left(M_{1}, M_{2}\right) \otimes \mathcal{D}_{s}\left(M_{1}\right) & \rightarrow \mathcal{D}_{s-r}\left(M_{2}\right), \\
(f, z) & \mapsto f \cap z,
\end{aligned}
$$

as follows.

Let $F_{*}\left(M_{i}\right)$ be a free resolution of $M_{i}, i=1,2$. A class $f \in \operatorname{Ext}_{\mathcal{A}}^{r}\left(M_{1}, M_{2}\right)$ can be represented by a chain map $F: F_{*}\left(M_{1}\right) \rightarrow F_{*-r}\left(M_{2}\right)$ of homological degree $-r$. We write $f=[F]$. If $z=[Z]$ is represented by $Z \in F_{*}\left(M_{1}\right)$, then by definition $f \cap z=[F(Z)]$.

Let $M$ be an $\mathcal{A}$-module. We set $r=s=k, M_{1}=\Sigma^{-k} M, M_{2}=P_{k} \otimes M$, where as before $P_{k}=\mathbb{F}_{2}\left[x_{1}, \ldots, x_{k}\right]$, and get the homomorphism

$$
\cap: \operatorname{Ext}_{\mathcal{A}}^{k}\left(\Sigma^{-k} M, P_{k} \otimes M\right) \otimes \mathcal{D}_{k}\left(\Sigma^{-k} M\right) \rightarrow P_{k} \otimes M .
$$

Now we need to define the Singer element $e_{k}(M) \in \operatorname{Ext}_{\mathcal{A}}^{k}\left(\Sigma^{-k} M, P_{k} \otimes M\right)$ (see Singer $\left[20\right.$, p. 498]). Let $\widehat{P}_{1}$ be the submodule of $\mathbb{F}_{2}\left[x, x^{-1}\right]$ spanned by all powers $x^{i}$ with $i \geq-1$, where $|x|=1$. The $\mathcal{A}$-module structure on $\mathbb{F}_{2}\left[x, x^{-1}\right]$ extends that of $P_{1}=\mathbb{F}_{2}[x]$ (see Adams [2], Wilkerson [22]). The inclusion $P_{1} \subset \widehat{P}_{1}$ gives rise to a short exact sequence of $\mathcal{A}$-modules:

$$
0 \rightarrow P_{1} \rightarrow \widehat{P}_{1} \rightarrow \Sigma^{-1} \mathbb{F}_{2} \rightarrow 0 .
$$

Denote by $e_{1}$ the corresponding element in $\operatorname{Ext}_{\mathcal{A}}^{1}\left(\Sigma^{-1} \mathbb{F}_{2}, P_{1}\right)$.

Definition 2.2. (Singer [20]).

(i) $e_{k}=\underbrace{e_{1} \otimes \cdots \otimes e_{1}}_{k \text { times }} \in \operatorname{Ext}_{\mathcal{A}}^{k}\left(\Sigma^{-k} \mathbb{F}_{2}, P_{k}\right)$.

(ii) $e_{k}(M)=e_{k} \otimes M \in \operatorname{Ext}_{\mathcal{A}}^{k}\left(\Sigma^{-k} M, P_{k} \otimes M\right)$, for $M$ an $\mathcal{A}$-module.

Here we also denote by $M$ the identity map of $M$.

The cap-product with $e_{k}(M)$ gives rise to the homomorphism

$$
\begin{array}{cccc}
e_{k}(M): \mathcal{D}_{k}\left(\Sigma^{-k} M\right) & \rightarrow & \mathcal{D}_{0}\left(P_{k} \otimes M\right) \equiv P_{k} \otimes M, \\
e_{k}(M)(z) & = & e_{k}(M) \cap z .
\end{array}
$$

As $\mathbb{F}_{2}$ is an unstable $\mathcal{A}$-module, the following theorem is a special case (but would be the most important case) of the main result in [13].

Theorem 2.3 (Lannes-Zarati [13]). Let $D_{k} \subset P_{k}$ be the Dickson algebra of $k$ variables. Then $e_{k}\left(\Sigma \mathbb{F}_{2}\right): \mathcal{D}_{k}\left(\Sigma^{1-k} \mathbb{F}_{2}\right) \rightarrow \Sigma D_{k}$ is an isomorphism of internal degree 0 .

Next, we explain in detail the definition of the Lannes-Zarati homomorphism

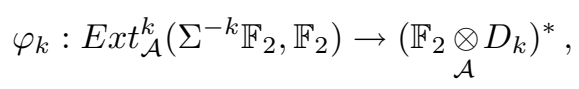

which is compatible with the Hurewicz map (see [12], [13]).

Let $N$ be an $\mathcal{A}$-module. By definition of the functor $\mathcal{D}$, we have a natural homomorphism: $\mathcal{D}(N) \rightarrow \mathbb{F}_{2} \otimes N$. Suppose $F_{*}(N)$ is a free resolution of $N$. Then the above natural homomorphism induces a commutative diagram 


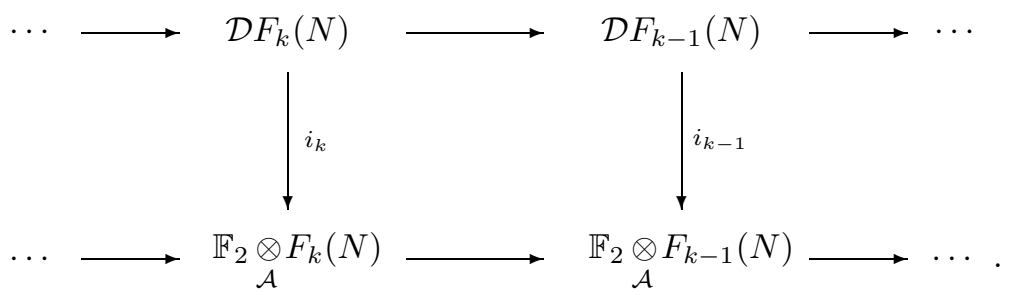

Here the horizontal arrows are induced from the differential in $F_{*}(N)$, and

$$
i_{k}[Z]=[1 \underset{\mathcal{A}}{\otimes} Z]
$$

for $Z \in F_{k}(N)$. Passing to homology, we get a homomorphism

$$
\begin{array}{cccc}
i_{k}: & \mathbb{F}_{2} \otimes \mathcal{D}_{k}(N) & \rightarrow & \operatorname{Tor}_{k}^{\mathcal{A}}\left(\mathbb{F}_{2}, N\right), \\
& \mathcal{A}_{\mathcal{A}} \otimes[Z] & \mapsto & {[1 \underset{\mathcal{A}}{\otimes} Z] .}
\end{array}
$$

Taking $N=\Sigma^{1-k} \mathbb{F}_{2}$, we obtain a homomorphism

$$
i_{k}: \mathbb{F}_{2} \underset{\mathcal{A}}{\otimes \mathcal{D}_{k}}\left(\Sigma^{1-k} \mathbb{F}_{2}\right) \rightarrow \operatorname{Tor}_{k}^{\mathcal{A}}\left(\mathbb{F}_{2}, \Sigma^{1-k} \mathbb{F}_{2}\right) .
$$

Note that the suspension $\Sigma: \mathbb{F}_{2} \otimes D_{\mathcal{A}} \rightarrow \mathbb{F}_{2} \underset{\mathcal{A}}{\otimes} D_{k}$ and the desuspension

$$
\Sigma^{-1}: \operatorname{Tor}_{k}^{\mathcal{A}}\left(\mathbb{F}_{2}, \Sigma^{1-k} \mathbb{F}_{2}\right) \stackrel{\cong}{\longrightarrow} \operatorname{Tor}_{k}^{\mathcal{A}}\left(\mathbb{F}_{2}, \Sigma^{-k} \mathbb{F}_{2}\right)
$$

are isomorphisms of internal degree 1 and $(-1)$, respectively. This leads us to

Definition 2.4. (Lannes-Zarati [13]). The homomorphism $\varphi_{k}$ of internal degree 0 is the dual of

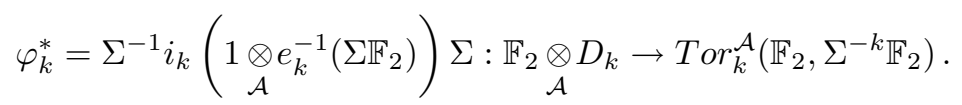

Now we recall the definition of the algebraic transfer. Consider the cap-product

$$
\begin{array}{ccc}
\operatorname{Ext}_{\mathcal{A}}^{r}\left(\Sigma^{-k} \mathbb{F}_{2}, P_{k}\right) \otimes \operatorname{Tor}_{s}^{\mathcal{A}}\left(\mathbb{F}_{2}, \Sigma^{-k} \mathbb{F}_{2}\right) & \rightarrow & \operatorname{Tor}_{s-r}^{\mathcal{A}}\left(\mathbb{F}_{2}, P_{k}\right), \\
(e, z) & \mapsto & e \cap z .
\end{array}
$$

Taking $r=s=k$ and $e=e_{k}$ as in Definition 2.2, we obtain the homomorphism

$$
\begin{aligned}
& \operatorname{Tr}_{k}^{*}: \operatorname{Tor}_{k}^{\mathcal{A}}\left(\mathbb{F}_{2}, \Sigma^{-k} \mathbb{F}_{2}\right) \rightarrow \operatorname{Tor}_{0}^{\mathcal{A}}\left(\mathbb{F}_{2}, P_{k}\right) \equiv \mathbb{F}_{2} \otimes P_{k},
\end{aligned}
$$

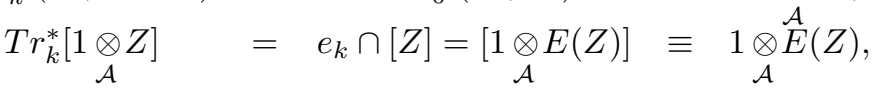

for $Z \in F_{k}\left(\Sigma^{-k} \mathbb{F}_{2}\right)$, where $e_{k}=[E]$ is represented by a chain map $E: F_{*}\left(\Sigma^{-k} \mathbb{F}_{2}\right) \rightarrow$ $F_{*-k}\left(P_{k}\right)$.

Singer proved in [20] that $e_{k}$ is $G L_{k}$-invariant, hence $\operatorname{Im}\left(\operatorname{Tr}_{k}^{*}\right) \subset\left(\mathbb{F}_{2} \underset{\mathcal{A}}{\otimes} P_{k}\right)^{G L_{k}}$. This gives rise to a homomorphism, which is also denoted by $\operatorname{Tr}_{k}^{*}$,

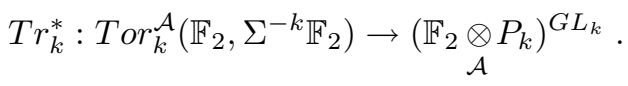

Definition 2.5. (Singer [20]). The $k$-th algebraic transfer $T r_{k}: \mathbb{F}_{2} \underset{G L_{k}}{\otimes} P H_{*}\left(B V_{k}\right)$ $\rightarrow \operatorname{Ext}_{\mathcal{A}}^{k, k+*}\left(\mathbb{F}_{2}, \mathbb{F}_{2}\right)$ is the homomorphism dual to $\operatorname{Tr}_{k}^{*}$.

We have finished the preparation of the needed data. 
Proof of Theorem 2.1. Note that the usual isomorphism

$$
\operatorname{Ext}_{\mathcal{A}}^{k}\left(\Sigma^{-k} \mathbb{F}_{2}, P_{k}\right) \stackrel{\cong}{\longrightarrow} \operatorname{Ext}_{\mathcal{A}}^{k}\left(\Sigma^{1-k} \mathbb{F}_{2}, \Sigma P_{k}\right)
$$

sends $e_{k}\left(\mathbb{F}_{2}\right)$ to $e_{k}\left(\Sigma \mathbb{F}_{2}\right)=e_{k}\left(\mathbb{F}_{2}\right) \otimes \Sigma \mathbb{F}_{2}$. Moreover, if $e_{k}\left(\mathbb{F}_{2}\right)=[E]$ is represented by a chain map $E: F_{*}\left(\Sigma^{-k} \mathbb{F}_{2}\right) \rightarrow F_{*-k}\left(P_{k}\right)$ then $e_{k}\left(\Sigma \mathbb{F}_{2}\right)=\left[E_{\Sigma}\right]$ is represented by the induced chain map $E_{\Sigma}: F_{*}\left(\Sigma^{1-k} \mathbb{F}_{2}\right) \rightarrow F_{*-k}\left(\Sigma P_{k}\right)$, which is defined by $E_{\Sigma}=\Sigma E \Sigma^{-1}$.

By Theorem 2.3, $e_{k}\left(\Sigma \mathbb{F}_{2}\right)$ is an isomorphism. So, for any $Y \in D_{k}$, there exists a representative of $e_{k}^{-1}\left(\Sigma \mathbb{F}_{2}\right) \Sigma Y$, which is denoted by $E_{\Sigma}^{-1} \Sigma Y \in F_{k}\left(\Sigma^{1-k} \mathbb{F}_{2}\right)$, such that $E_{\Sigma}\left(E_{\Sigma}^{-1} \Sigma Y\right)=\Sigma Y$.

The cap-product with $e_{k}\left(\Sigma \mathbb{F}_{2}\right)=\left[E_{\Sigma}\right]$ induces the homomorphism

$$
\begin{aligned}
& \widetilde{T r}_{k}^{*}: \operatorname{Tor}_{k}^{\mathcal{A}}\left(\mathbb{F}_{2}, \Sigma^{1-k} \mathbb{F}_{2}\right) \rightarrow \operatorname{Tor}_{0}^{\mathcal{A}}\left(\mathbb{F}_{2}, \Sigma P_{k}\right) \quad \equiv \mathbb{F}_{2} \otimes \Sigma P_{k},
\end{aligned}
$$

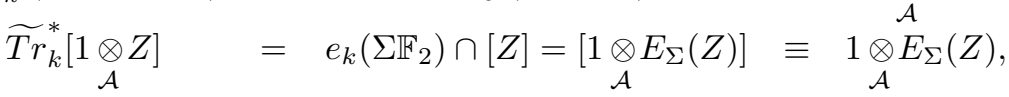

for $Z \in F_{k}\left(\Sigma^{1-k} \mathbb{F}_{2}\right)$. It is easy to check that $\operatorname{Tr}_{k}^{*}=\Sigma^{-1} \widetilde{T r}_{k}^{*} \Sigma$. Moreover, set

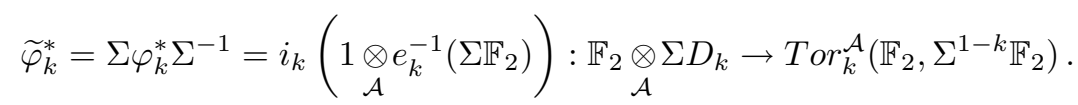

Obviously, $\operatorname{Tr}_{k}^{*} \cdot \varphi_{k}^{*}=\Sigma^{-1} \widetilde{\operatorname{Tr}}_{k}^{*} \cdot \widetilde{\varphi}_{k}^{*} \Sigma$. Now, for any $Y \in D_{k}$, we have

$$
\begin{aligned}
& \operatorname{Tr}_{k}^{*} \cdot \varphi_{k}^{*}(1 \underset{\mathcal{A}}{\otimes Y})=\Sigma^{-1} \widetilde{T r}_{k}^{*} \cdot \widetilde{\varphi}_{k}^{*} \Sigma(\underset{\mathcal{A}}{\otimes} Y) \\
& =\Sigma^{-1} \widetilde{\operatorname{Tr}}_{k}^{*} \cdot \widetilde{\varphi}_{k}^{*}(1 \underset{\mathcal{A}}{\otimes \Sigma Y}) \\
& =\Sigma^{-1} \widetilde{\operatorname{Tr}}_{k}^{*}\left[\underset{\mathcal{A}}{\left.\otimes E_{\Sigma}^{-1} \Sigma Y\right]}\right. \\
& =\Sigma^{-1}\left(\underset{\mathcal{A}}{1 \otimes E_{\Sigma}}\left(E_{\Sigma}^{-1} \Sigma Y\right)\right) \\
& =1 \otimes \Sigma^{-1}(\Sigma Y) \\
& =\stackrel{\mathcal{A}}{\otimes} Y \text {. }
\end{aligned}
$$

By definition of $j_{k}$, we also have $j_{k}(1 \underset{\mathcal{A}}{\otimes} Y)=1 \underset{\mathcal{A}}{\otimes} Y$. The theorem is proved.

\section{The homomorphism of COALgebras $j=\bigoplus j_{k}$}

The canonical isomorphism $V_{k} \cong V_{\ell} \times V_{m}$, for $k=\ell+m$, induces the usual inclusion $G L_{k} \supset G L_{\ell} \times G L_{m}$ and the usual diagonal $\Delta: P_{k} \rightarrow P_{\ell} \otimes P_{m}$. Therefore, it induces two homomorphisms

$$
\begin{aligned}
& \bar{\Delta}_{D}: \mathbb{F}_{2} \underset{\mathcal{A}}{\otimes}\left(P_{k}^{G L_{k}}\right) \rightarrow\left(\mathbb{F}_{2} \stackrel{\mathcal{A}}{\otimes}_{\left(P_{\ell}^{G L_{\ell}}\right)}\right) \otimes\left(\mathbb{F}_{2} \stackrel{\mathcal{A}}{\otimes}_{\left(P^{G}\right.}\left(P_{m}^{G L_{m}}\right)\right), \\
& \bar{\Delta}_{P}:\left(\mathbb{F}_{2} \otimes P_{\mathcal{A}}\right)^{G L_{k}} \rightarrow\left(\mathbb{F}_{2} \otimes P_{\mathcal{A}} P^{G L_{\ell}} \otimes \underset{\mathcal{A}}{\left(\mathbb{F}_{2} \otimes P_{m}\right.}\right)^{G L_{m}} .
\end{aligned}
$$

Here and in what follows, $\otimes$ means the tensor product over $\mathbb{F}_{2}$, except when otherwise specified. 
Set

$$
\begin{aligned}
& \mathbb{F}_{2} \underset{\mathcal{A}}{\otimes} D=\mathbb{F}_{2} \underset{\mathcal{A}}{\otimes}\left(P^{G L}\right):=\bigoplus_{k \geq 0} \mathbb{F}_{2} \underset{\mathcal{A}}{\otimes}\left(P_{k}^{G L_{k}}\right) \\
& \left(\mathbb{F}_{2} \otimes P\right)^{G L}:=\bigoplus_{k \geq 0}\left(\mathbb{F}_{2} \otimes P_{\mathcal{A}} P_{k}\right)^{G L_{k}} .
\end{aligned}
$$

It is easy to see that $\mathbb{F}_{2} \otimes\left(P^{G L}\right)$ and $\left(\mathbb{F}_{2} \otimes P\right)^{G L}$ are endowed with the structure of a cocommutative coalgebra by $\bar{\Delta}_{D}$ and $\mathcal{A}_{P}$, respectively. The coalgebra structure of $\left(\mathbb{F}_{2} \underset{\mathcal{A}}{\otimes} P\right)^{G L}$ was first given by Singer [20].

Proposition 3.1. $j=\bigoplus j_{k}: \mathbb{F}_{2} \underset{\mathcal{A}}{\otimes}\left(P^{G L}\right) \rightarrow\left(\mathbb{F}_{2} \underset{\mathcal{A}}{\otimes} P\right)^{G L}$ is a homomorphism of coalgebras.

Proof. This follows immediately from the commutative diagram

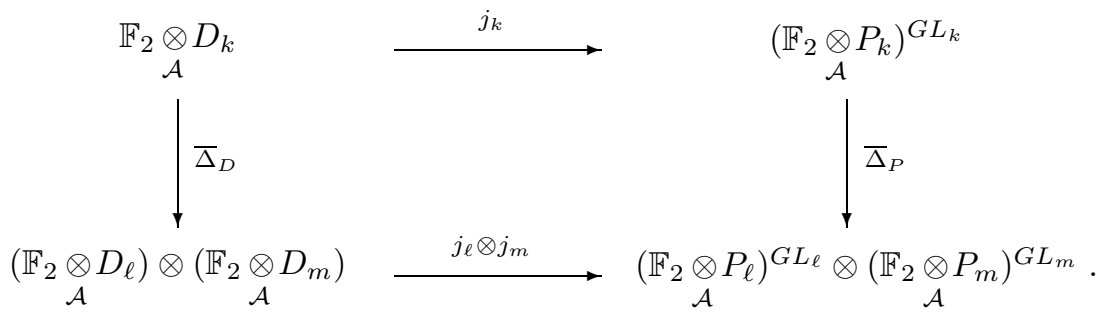

Remark. According to Singer [20], $T r^{*}=\bigoplus T r_{k}^{*}$ is a homomorphism of coalgebras. One can see that $\varphi^{*}=\bigoplus \varphi_{k}^{*}$ is also a homomorphism of coalgebras. Then, so is $j=T r^{*} \cdot \varphi^{*}$. This is an alternative proof for Proposition 3.1.

Now let

$$
\begin{gathered}
\mathbb{F}_{2} \underset{G L}{\otimes} P H_{*}(B V):=\bigoplus_{k \geq 0}\left(\mathbb{F}_{2} \underset{G L_{k}}{\otimes} P H_{*}\left(B V_{k}\right)\right) \cong \bigoplus_{k \geq 0}\left(\underset{\mathcal{A}}{\left.\left(\mathbb{F}_{2} \underset{P_{k}}{\otimes}\right)^{G L_{k}}\right)^{*},}\right. \\
P\left(\mathbb{F}_{2} \underset{G L}{\otimes} H_{*}(B V)\right):=\bigoplus_{k \geq 0} P\left(\mathbb{F}_{2} \underset{G L_{k}}{\otimes} H_{*}\left(B V_{k}\right)\right) \cong \bigoplus_{k \geq 0}\left(\mathbb{F}_{2} \underset{\mathcal{A}}{\otimes}\left(P_{k}^{G L_{k}}\right)\right)^{*} .
\end{gathered}
$$

Passing to the dual, we obtain the homomorphism of algebras

$$
j^{*}: \mathbb{F}_{2} \underset{G L}{\otimes} P H_{*}(B V) \rightarrow P\left(\mathbb{F}_{2} \underset{G L}{\otimes} H_{*}(B V)\right) .
$$

As an application of $j^{*}$, we give here a proof for Conjecture 1.4 with $k=3$.

Theorem 3.2. $j_{3}: \mathbb{F}_{2} \underset{\mathcal{A}}{\otimes}\left(P_{3}^{G L_{3}}\right) \rightarrow\left(\mathbb{F}_{2} \otimes P_{\mathcal{A}} P_{3}\right)^{G L_{3}}$ is zero in positive dimensions.

Proof. We equivalently show that

$$
j_{3}^{*}: \mathbb{F}_{2} \underset{G L_{3}}{\otimes} P H_{*}\left(B V_{3}\right) \rightarrow P\left(\mathbb{F}_{2} \underset{G L_{3}}{\otimes} H_{*}\left(B V_{3}\right)\right)
$$

is a trivial homomorphism in positive dimensions.

$\mathbb{F}_{2} \otimes P H_{*}\left(B V_{3}\right)$ is described by Kameko [11], Alghamdi-Crabb-Hubbuck [3] $G L_{3}$ and Boardman [4] as follows. $\mathbb{F}_{2} \otimes P H_{*}\left(B V_{1}\right)$ has a basis consisting of $h_{r}, r \geq$ 0 , where $h_{r}$ is of dimension $2^{r}-1$ and is sent by the isomorphism $\operatorname{Tr}_{1}$ to the Adams element, denoted also by $h_{r}$, in $\operatorname{Ext}_{\mathcal{A}}^{1,2^{r}}\left(\mathbb{F}_{2}, \mathbb{F}_{2}\right)$. According to [11], [3], 
[4], $\mathbb{F}_{2} \otimes P H_{*}\left(B V_{3}\right)$ has a basis consisting of some products of the form $h_{r} h_{s} h_{t}$, $G L_{3}$ where $r, s, t$ are non-negative integers (but not all such appear), and some elements $c_{i}(i \geq 0)$ with $\operatorname{dim}\left(c_{i}\right)=2^{i+3}+2^{i+1}+2^{i}-3$.

We will show in Lemma 3.3 that any decomposable element in $P\left(\mathbb{F}_{2} \otimes H_{*}\left(B V_{3}\right)\right)$ is zero. Then, since $j^{*}$ is a homomorphism of algebras, $j_{3}^{*}$ sends any element of the form $h_{r} h_{s} h_{t}$ to zero.

On the other hand, by Hu'ng-Peterson [18], $\mathbb{F}_{2} \otimes D_{3}$ is concentrated in the dimensions $2^{s+2}-4(s \geq 0)$ and $\left.2^{r+2}+2^{s+1}-3 \stackrel{\mathcal{A}}{(r}>s>0\right)$. Obviously, these dimensions are different from $\operatorname{dim}\left(c_{i}\right)$ for any $i$. Then $j_{3}^{*}$ also sends $c_{i}$ to zero.

To complete the proof of the theorem, we need to show the following lemma.

Lemma 3.3. Let $\bar{D}_{k}=\mathbb{F}_{2} \otimes_{\mathcal{A}} D_{k}$. Then the diagonal

$$
\bar{\Delta}_{D}: \bar{D}_{3} \rightarrow \bar{D}_{1} \otimes \bar{D}_{2} \oplus \bar{D}_{2} \otimes \bar{D}_{1}
$$

is zero in positive dimensions.

Proof. Let us recall some informations on the Dickson algebra $D_{k}$. Dickson proved in [10] that $D_{k} \cong \mathbb{F}_{2}\left[Q_{k-1}, Q_{k-2}, \ldots, Q_{0}\right]$, a polynomial algebra on $k$ generators, with $\left|Q_{s}\right|=2^{k}-2^{s}$. Note that $Q_{s}$ depends on $k$, and when necessary, will be denoted $Q_{k, s}$. An inductive definition of $Q_{k, s}$ is given by

\section{Definition 3.4.}

$$
Q_{k, s}=Q_{k-1, s-1}^{2}+v_{k} \cdot Q_{k-1, s},
$$

where, by convention, $Q_{k, k}=1, Q_{k, s}=0$ for $s<0$ and

$$
v_{k}=\prod_{\lambda_{i} \in \mathbb{F}_{2}}\left(\lambda_{1} x_{1}+\cdots+\lambda_{k-1} x_{k-1}+x_{k}\right) .
$$

Dickson showed in [10] that

$$
v_{k}=\sum_{s=0}^{k-1} Q_{k-1, s} x_{k}^{2^{s}} .
$$

Now we turn back to the lemma.

Since $\bar{\Delta}_{D}$ is symmetric, we need only to show that the diagonal

$$
\bar{\Delta}: \bar{D}_{3} \rightarrow \bar{D}_{2} \otimes \bar{D}_{1}
$$

is zero in positive dimensions.

For abbreviation, we denote $x_{1}, x_{2}, x_{3}$ by $x, y, z$, respectively, $Q_{i}=Q_{3, i}(x, y, z)$ for $i=0,1,2, q_{i}=Q_{2, i}(x, y)$ for $i=0,1$. As is well known, $\mathbb{F}_{2} \otimes D_{1}$ has the basis $\left\{z^{2^{s}-1} \mid s \geq 0\right\}$, and $\mathbb{F}_{2} \otimes D_{2}$ has the basis $\left\{q_{1}^{2^{s}-1} \mid s \geq 0\right\}$. By Hu'ng-Peterson [18], $\mathbb{F}_{2} \otimes D_{3}$ has the basis

$\mathcal{A}$

$$
\left\{Q_{2}^{2^{s}-1}(s \geq 0), Q_{2}^{2^{r}-2^{s}-1} Q_{1}^{2^{s}-1} Q_{0}(r>s>0)\right\} .
$$

For $k \leq 3$, every monomial in $Q_{0}, \ldots, Q_{k-1}$ which does not belong to the given basis is zero in $\mathbb{F}_{2} \otimes D_{\mathcal{A}}$. Note that the analogous statement is not true for $k \geq 4$ (see $[18])$. 
Using the above inductive definitions of $Q_{k, s}$ and $v_{k}$, we get

$$
Q_{0}=q_{0}^{2} z+q_{0} q_{1} z^{2}+q_{0} z^{4},
$$

or

$$
\Delta\left(Q_{0}\right)=q_{0}^{2} \otimes z+q_{0} q_{1} \otimes z^{2}+q_{0} \otimes z^{4} .
$$

This implies easily that every term in $\Delta\left(Q_{2}^{2^{r}-2^{s}-1} Q_{1}^{2^{s}-1} Q_{0}\right)$ is divisible by $q_{0}$, so it equals zero in $\mathbb{F}_{2} \otimes D_{2}$ as shown above. In other words,

$$
\bar{\Delta}\left(Q_{2}^{2^{r}-2^{s}-1} Q_{1}^{2^{s}-1} Q_{0}\right)=0
$$

Similarly,

$$
Q_{2}=q_{1}^{2}+v_{3}=q_{1}^{2}+q_{0} z+q_{1} z^{2}+z^{4}
$$

or

$$
\begin{aligned}
& \Delta\left(Q_{2}\right)=q_{1}^{2} \otimes 1+q_{0} \otimes z+q_{1} \otimes z^{2}+1 \otimes z^{4}, \\
& \Delta\left(Q_{2}^{2^{s}-1}\right)=\left(q_{1}^{2} \otimes 1+q_{0} \otimes z+q_{1} \otimes z^{2}+1 \otimes z^{4}\right)^{2^{s}-1} .
\end{aligned}
$$

By the same argument as above, we need only to consider terms in $\Delta\left(Q_{2}^{2^{s}-1}\right)$ which are not divisible by $q_{0}$. Such a term is some product of powers of $q_{1}^{2} \otimes 1, q_{1} \otimes z^{2}$, $1 \otimes z^{4}$. If it contains a positive power of $z$ then this power is even and it equals zero in $\mathbb{F}_{2} \otimes D_{1}$. Otherwise, it should be $q_{1}^{2\left(2^{s}-1\right)} \otimes 1$. Obviously, $q_{1}^{2\left(2^{s}-1\right)}$ equals zero in

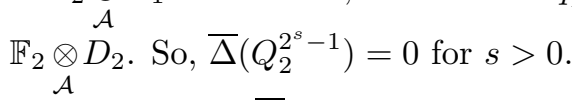

In summary, $\bar{\Delta}=0$ in positive dimensions. The lemma is proved. Then, so is Theorem 3.2.

As $\operatorname{Tr}_{3}$ is an isomorphism (see Boardman [4]), we have an immediate consequence.

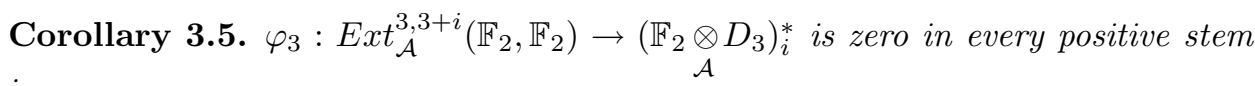
$i$.

\section{The SQUARING OPERATION: THE EXISTENCE}

Liulevicius was perhaps the first person who noted in [14] that there are squaring operations $S q^{i}: \operatorname{Ext}_{\mathcal{A}}^{k, t}\left(\mathbb{F}_{2}, \mathbb{F}_{2}\right) \rightarrow \operatorname{Ext}_{\mathcal{A}}^{k+i, 2 t}\left(\mathbb{F}_{2}, \mathbb{F}_{2}\right)$, which share most of the properties with $S q^{i}$ on cohomology of spaces. In particular, $S q^{i}(\alpha)=0$ if $i>k$, $S q^{k}(\alpha)=\alpha^{2}$ for $\alpha \in \operatorname{Ext}_{\mathcal{A}}^{k, t}\left(\mathbb{F}_{2}, \mathbb{F}_{2}\right)$, and the Cartan formula holds for the $S q^{i}$ 's. However, $S q^{0}$ is not the identity. In fact,

$$
\begin{aligned}
& S q^{0}: \operatorname{Ext}_{\mathcal{A}}^{k, t}\left(\mathbb{F}_{2}, \mathbb{F}_{2}\right) \rightarrow \operatorname{Ext}_{\mathcal{A}}^{k, 2 t}\left(\mathbb{F}_{2}, \mathbb{F}_{2}\right), \\
& {\left[b_{1}|\ldots| b_{k}\right] \quad \mapsto \quad\left[b_{1}^{2}|\ldots| b_{k}^{2}\right],}
\end{aligned}
$$

in terms of the cobar resolution (see May [16]).

Recall that $H_{*}\left(B V_{k}\right)$ is a divided power algebra

$$
H_{*}\left(B V_{k}\right)=\Gamma\left(a_{1}, \ldots, a_{k}\right)
$$

generated by $a_{1}, \ldots, a_{k}$, each of degree 1 , where $a_{i}$ is dual to $x_{i} \in H^{1}\left(B V_{k}\right)$. Here and in what follows, the duality is taken with respect to the basis of $H^{*}\left(B V_{k}\right)$ consisting of all monomials in $x_{1}, \ldots, x_{k}$. 
Let $\gamma_{t}$ be the $t$-th divided power in $H_{*}\left(B V_{k}\right)$ and for any $a \in H_{*}\left(B V_{k}\right)$ let $a^{(t)}=\gamma_{t}(a)$. So $a_{i}^{(t)}$ is the element dual to $x_{i}^{t}$. One has

$$
a_{i}^{\left(2^{r}\right)} a_{i}^{\left(2^{r}\right)}=0,
$$

and

$$
a_{i}^{(t)}=a_{i}^{\left(2^{r_{1}}\right)} \cdots a_{i}^{\left(2^{r_{m}}\right)}
$$

if $t=2^{r_{1}}+\cdots+2^{r_{m}}, 0 \leq r_{1}<\cdots<r_{m}$.

In [11] Kameko defined a $G L_{k}$-homomorphism

$$
\begin{aligned}
& S q^{0}: P H_{*}\left(B V_{k}\right) \quad \rightarrow \quad P H_{*}\left(B V_{k}\right), \\
& a_{1}^{\left(i_{1}\right)} \cdots a_{k}^{\left(i_{k}\right)} \mapsto a_{1}^{\left(2 i_{1}+1\right)} \cdots a_{k}^{\left(2 i_{k}+1\right)},
\end{aligned}
$$

where $a_{1}^{\left(i_{1}\right)} \cdots a_{k}^{\left(i_{k}\right)}$ is dual to $x_{1}^{i_{1}} \cdots x_{k}^{i_{k}}$. (See also [3].)

Crabb and Hubbuck gave in [8] a definition of $S q^{0}$ that does not depend on the chosen basis of $H_{*}\left(B V_{k}\right)$ as follows. The element $a\left(V_{k}\right)=a_{1} \cdots a_{k}$ is nothing but the image of the generator of $\Lambda^{k}\left(V_{k}\right)$ under the (skew) symmetrization map

$$
\Lambda^{k}\left(V_{k}\right) \rightarrow H_{k}\left(B V_{k}\right)=\Gamma_{k}\left(V_{k}\right)=(\underbrace{V_{k} \otimes \cdots \otimes V_{k}}_{k \text { times }})_{\Sigma_{k}} .
$$

Let $F: H^{*}\left(B V_{k}\right) \rightarrow H^{*}\left(B V_{k}\right)$ be the Frobenius homomorphism defined by $F(x)=$ $x^{2}$ for any $x$, and let $c: H_{*}\left(B V_{k}\right) \rightarrow H_{*}\left(B V_{k}\right)$ be the degree-halving dual homomorphism. It is obviously a surjective ring homomorphism. Then $S q^{0}$ can be defined by

$$
S q^{0}(c(y))=a\left(V_{k}\right) y .
$$

Since $y \in \operatorname{ker} c$ if and only if $a\left(V_{k}\right) y=0, S q^{0}$ is a monomorphism of $G L_{k}$-modules. Further, it is easy to see that $c S q_{*}^{2 i+1}=0, c S q_{*}^{2 i}=S q_{*}^{i} c$. So $S q^{0}$ maps $P H_{*}\left(B V_{k}\right)$ to itself.

Using a result of Carlisle and Wood [6] on the boundedness conjecture, Crabb and Hubbuck also noted in [8] that for any $d$, there exists $t_{0}$ such that

$$
S q^{0}: P H_{2^{t} d+\left(2^{t}-1\right) k}\left(B V_{k}\right) \rightarrow P H_{2^{t+1} d+\left(2^{t+1}-1\right) k}\left(B V_{k}\right)
$$

is an isomorphism for every $t \geq t_{0}$.

Kameko's $S q^{0}$ is shown to commute with $S q^{0}$ on $\operatorname{Ext}_{\mathcal{A}}^{k}\left(\mathbb{F}_{2}, \mathbb{F}_{2}\right)$ through the algebraic transfer $T r_{k}$ by Boardman [4] for $k=3$ and by Minami [17] for general $k$.

One denotes also by $S q^{0}$ the operation

$$
S q^{0}: \mathbb{F}_{2} \underset{G L_{k}}{\otimes} P H_{*}\left(B V_{k}\right) \rightarrow \mathbb{F}_{2} \underset{G L_{k}}{\otimes} P H_{*}\left(B V_{k}\right)
$$

induced by Kameko's $S q^{0}$. It preserves the product. Further, for $k=3$, it satisfies

$$
S q^{0}\left(h_{r} h_{s} h_{t}\right)=h_{r+1} h_{s+1} h_{t+1}, \quad S q^{0}\left(c_{i}\right)=c_{i+1}
$$

(see Boardman [4]).

Lemma 4.1. $S q_{*}^{2 r+1} S q^{0}=0, S q_{*}^{2 r} S q^{0}=S q^{0} S q_{*}^{r}$.

Proof. We need a formal notation. Namely, for $a \in H_{1}\left(B V_{k}\right)$, set $\left(a^{(t)}\right)^{[2]}=a^{(2 t)}$. In general, $\left(a^{(t)}\right)^{[2]} \neq \gamma_{2}\left(\gamma_{t}(a)\right)=\left(\begin{array}{c}2 t-1 \\ t\end{array}\right) a^{(2 t)}$ (see Cartan [7]). 
We start with a simple remark.

Let $x \in H^{1}\left(B V_{k}\right)$; then $S q^{r}\left(x^{s}\right)=\left(\begin{array}{l}s \\ r\end{array}\right) x^{s+r}$. Let $a$ denote the dual element of $x$. Then, by dualizing,

$$
S q_{*}^{r}\left(a^{(t)}\right)=\left(\begin{array}{c}
t-r \\
r
\end{array}\right) a^{(t-r)} .
$$

As a consequence, $S q_{*}^{2 r+1}\left(a^{(2 t+1)}\right)=0$ and

$$
S q_{*}^{2 r}\left(a^{(2 t)}\right)=\left(\begin{array}{c}
2 t-2 r \\
2 r
\end{array}\right) a^{(2 t-2 r)}=\left(\begin{array}{c}
t-r \\
r
\end{array}\right)\left(a^{(t-r)}\right)^{[2]}=\left(S q_{*}^{r} a^{(t)}\right)^{[2]} .
$$

Let $\alpha=a_{1}^{\left(i_{1}\right)} \cdots a_{k}^{\left(i_{k}\right)}$. By the Cartan formula, we have

$$
\begin{aligned}
S q_{*}^{r} S q^{0}(\alpha) & =S q_{*}^{r}\left(a_{1}^{\left(2 i_{1}+1\right)} \cdots a_{k}^{\left(2 i_{k}+1\right)}\right) \\
& =\sum_{r_{1}+\cdots+r_{k}=r} S q_{*}^{r_{1}}\left(a_{1}^{\left(2 i_{1}+1\right)}\right) \cdots S q_{*}^{r_{k}}\left(a_{k}^{\left(2 i_{k}+1\right)}\right) .
\end{aligned}
$$

The term corresponding to $\left(r_{1}, \ldots, r_{k}\right)$ equals 0 if at least one of $r_{1}, \ldots, r_{k}$ is odd. Hence $S q_{*}^{2 r+1} S q^{0}(\alpha)=0$. Furthermore,

$$
\begin{aligned}
S q_{*}^{2 r} S q^{0}(\alpha) & =\sum_{r_{1}+\cdots+r_{k}=r} S q_{*}^{2 r_{1}}\left(a_{1}^{\left(2 i_{1}+1\right)}\right) \cdots S q_{*}^{2 r_{k}}\left(a_{k}^{\left(2 i_{k}+1\right)}\right) \\
& =\sum_{r_{1}+\cdots+r_{k}=r}\left\{S q_{*}^{2 r_{1}}\left(a_{1}^{\left(2 i_{1}\right)}\right) \cdots S q_{*}^{2 r_{k}}\left(a_{k}^{\left(2 i_{k}\right)}\right)\right\} a_{1} \cdots a_{k} \\
& =\sum_{r_{1}+\cdots+r_{k}=r}\left\{S q_{*}^{r_{1}}\left(a_{1}^{\left(i_{1}\right)}\right)\right\}^{[2]} \cdots\left\{S q_{*}^{r_{k}}\left(a_{k}^{\left(i_{k}\right)}\right)\right\}^{[2]} a_{1} \cdots a_{k} \\
& =S q^{0} S q_{*}^{r}(\alpha) .
\end{aligned}
$$

The lemma is proved.

Proposition 4.2. For every positive integer $k$, there exists a homomorphism

$$
S q^{0}: P\left(\mathbb{F}_{2} \underset{G L_{k}}{\otimes} H_{*}\left(B V_{k}\right)\right) \rightarrow P\left(\mathbb{F}_{2} \underset{G L_{k}}{\otimes} H_{*}\left(B V_{k}\right)\right)
$$

that sends an element of degree $n$ to an element of degree $2 n+k$ and makes the following diagram commutative:

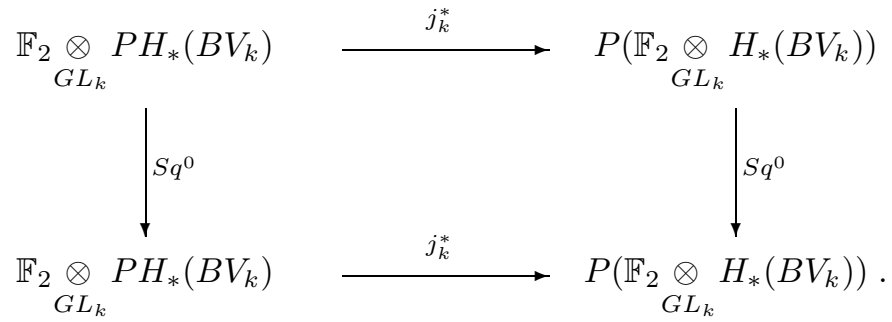

Proof. Since $S q^{0}: H_{*}\left(B V_{k}\right) \rightarrow H_{*}\left(B V_{k}\right)$ is a $G L_{k}$-homomorphism, we can define $S q_{D}^{0}=1 \otimes S q^{0}$ and get a commutative diagram $G L_{k}$ 


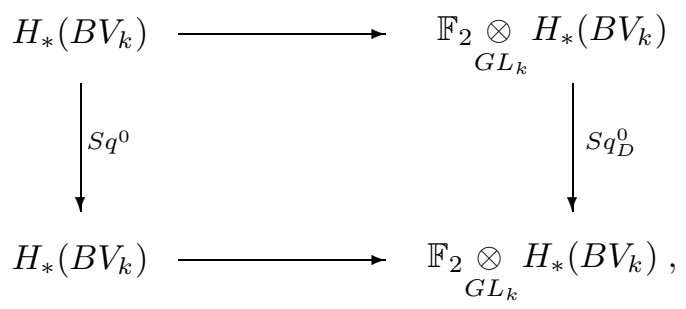

where the horizontal arrows are the canonical projections.

Next, we show that $S q_{D}^{0}$ sends the primitive part to itself. In other words, suppose $\alpha \in H_{*}\left(B V_{k}\right)$ satisfies

$$
S q_{*}^{r}\left(1 \underset{G L_{k}}{\otimes} \alpha\right)=1 \underset{G L_{k}}{\otimes} S q_{*}^{r} \alpha=0
$$

for any $r>0$; we want to show that

$$
S q_{*}^{r}\left(S q^{0}\left(\underset{G L_{k}}{\otimes} \alpha\right)\right)=0
$$

for any $r>0$. By definition of $S q^{0}$ and Lemma 4.1, we have for every $r>0$

$$
\begin{aligned}
& S q_{*}^{r}\left(S q^{0}\left(1 \underset{G L_{k}}{\otimes} \alpha\right)\right)=1 \underset{G L_{k}}{\otimes} S q_{*}^{r} S q^{0}(\alpha)
\end{aligned}
$$

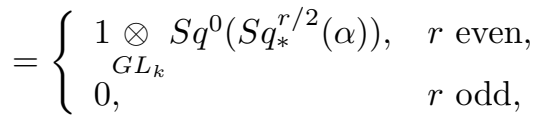

$$
\begin{aligned}
& = \begin{cases}S q^{0} S q_{*}^{r / 2}(1 \otimes \alpha), & r \text { even, } \\
0, & r \text { odd },\end{cases} \\
& =0 \text {. }
\end{aligned}
$$

Therefore, the above commutative diagram gives rise to a commutative diagram

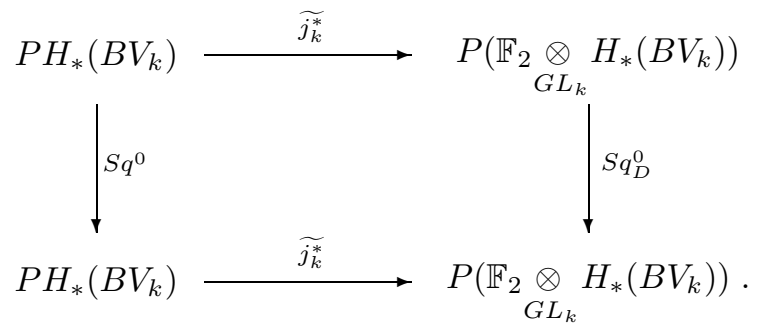

By definition of $j_{k}$, the homomorphism $\tilde{j_{k}^{*}}$ factors through $\mathbb{F}_{2} \underset{G L_{k}}{\otimes} P H_{*}\left(B V_{k}\right)$ and the previous diagram induces the commutative diagram stated in the proposition, in which $S q_{D}^{0}$ is re-denoted by $S q^{0}$ for short. The proposition is proved.

As an application of Proposition 4.2, we give an alternative proof of Theorem 3.2.

By Kameko [11], Alghamdi et al. [3] and Boardman [4], $\mathbb{F}_{2} \underset{G L_{3}}{\otimes} P H_{*}\left(B V_{3}\right)$ has a basis consisting of some products of the form $h_{r} h_{s} h_{t}$ and certain elements $c_{i}(i \geq 0)$ with $S q^{0}\left(c_{i}\right)=c_{i+1}$ for any $i \geq 0$.

By Lemma $3.3, j_{3}^{*}$ vanishes on any product $h_{r} h_{s} h_{t}$. Making use of Proposition 4.2 , one has

$$
j_{3}^{*}\left(c_{i}\right)=j_{3}^{*}\left(S q^{0}\right)^{i}\left(c_{0}\right)=\left(S q^{0}\right)^{i}\left(j_{3}^{*}\left(c_{0}\right)\right) .
$$


One needs only to show that $j_{3}^{*}\left(c_{0}\right)=0$. Recall that $\operatorname{dim}\left(c_{0}\right)=8$. The only element of dimension 8 in $D_{3}$ is $Q_{2}^{2}$. Obviously, $Q_{2}^{2}=S q^{4} Q_{2}$. So $P\left(\mathbb{F}_{2} \underset{G L_{3}}{\otimes} H_{*}\left(B V_{3}\right)\right)_{8}=$ $\left(\mathbb{F}_{2} \otimes D_{3}\right)_{8}^{*}=0$. Therefore, $j_{3}^{*}\left(c_{0}\right)=0$. Theorem 3.2 is proved.

5. THE SQUARING OPERATION: AN EXPLICIT FORMULA FOR $k \leq 4$

Let $d_{\left(i_{k-1}, \ldots, i_{0}\right)}$ be the dual element of $Q_{k-1}^{i_{k-1}} \cdots Q_{0}^{i_{0}} \in D_{k}$, where the duality is taken with respect to the basis of $D_{k}$ consisting of all monomials in the Dickson invariants $Q_{k-1}, \ldots, Q_{0}$.

It is well-known that

$$
\begin{aligned}
& P\left(\mathbb{F}_{2} \underset{G L_{1}}{\otimes} H_{*}\left(B V_{1}\right)\right)=\operatorname{Span}\left\{d_{\left(2^{s}-1\right)} \mid s \geq 0\right\}, \\
& P\left(\mathbb{F}_{2} \underset{G L_{2}}{\otimes} H_{*}\left(B V_{2}\right)\right)=\operatorname{Span}\left\{d_{\left(2^{s}-1,0\right)} \mid s \geq 0\right\} .
\end{aligned}
$$

By means of the definition of $S q^{0}$ one can easily show that

$$
\begin{aligned}
S q^{0}\left(d_{\left(2^{s}-1\right)}\right) & =d_{\left(2^{s+1}-1\right)}, \\
S q^{0}\left(d_{\left(2^{s}-1,0\right)}\right) & =d_{\left(2^{s+1}-1,0\right)} .
\end{aligned}
$$

In this section we compute $S q^{0}$ explicitly on $P\left(\mathbb{F}_{2} \underset{G L_{k}}{\otimes} H_{*}\left(B V_{k}\right)\right)$ for $k=3$ and 4 .

Theorem 5.1 (Hu'ng-Peterson [18]). $P D_{3}^{*}:=P\left(\mathbb{F}_{2} \underset{G L_{3}}{\otimes} H_{*}\left(B V_{3}\right)\right)$ has a basis consisting of

$$
\begin{aligned}
& d_{\left(2^{s}-1,0,0\right)}, \quad s \geq 0, \\
& d_{\left(2^{r}-2^{s}-1,2^{s}-1,1\right)}, \quad r>s>0 .
\end{aligned}
$$

They are of dimensions $2^{s+2}-4$ and $2^{r+2}+2^{s+1}-3$, respectively.

Remark. It is easy to check that $P D_{3}^{*}$ has at most one non-zero element of any dimension.

Proposition 5.2. $S q^{0}: P D_{3}^{*} \rightarrow P D_{3}^{*}$ is given by

$$
\begin{aligned}
& S q^{0}\left(d_{\left(2^{s}-1,0,0\right)}\right)=0, \\
& S q^{0}\left(d_{\left(2^{r}-2^{s}-1,2^{s}-1,1\right)}\right)=d_{\left(2^{r+1}-2^{s+1}-1,2^{s+1}-1,1\right)} .
\end{aligned}
$$

Proof. For brevity, we denote $x_{1}, x_{2}, x_{3}$ by $x, y, z$ and $a_{1}, a_{2}, a_{3}$ by $a, b, c$, respectively.

The first part of the proposition is an immediate consequence of dimensional information. To prove the second part we start by recalling that, from Definition 3.4, we have

$$
Q_{3,0}=Q_{0}=x^{4} y^{2} z^{1}+(\text { symmetrized }) .
$$

Suppose $m, n$ are non-negative integers. Let $x^{\alpha} y^{\beta} z^{\gamma}$ be the biggest monomial in $Q_{2}^{m} Q_{1}^{n}$ with respect to the lexicographic order on $(\alpha, \beta, \gamma)$. We claim that $x^{\alpha+4} y^{\beta+2} z^{\gamma+1}$ appears exactly one time in $Q_{2}^{m} Q_{1}^{n} Q_{0}$, or equivalently

$$
Q_{2}^{m} Q_{1}^{n} Q_{0}=x^{\alpha+4} y^{\beta+2} z^{\gamma+1}+\text { (other terms). }
$$


Indeed, suppose to the contrary that it appears more than once in $Q_{2}^{m} Q_{1}^{n} Q_{0}$. That means there exists a monomial $x^{\alpha^{\prime}} y^{\beta^{\prime}} z^{\gamma^{\prime}}$ in $Q_{2}^{m} Q_{1}^{n}$, which is different from $x^{\alpha} y^{\beta} z^{\gamma}$, and a permutation $\sigma$ on the set $\{4,2,1\}$ such that

$$
x^{\alpha+4} y^{\beta+2} z^{\gamma+1}=x^{\alpha^{\prime}+\sigma(4)} y^{\beta^{\prime}+\sigma(2)} z^{\gamma^{\prime}+\sigma(1)} .
$$

Since $\alpha+4=\alpha^{\prime}+\sigma(4)$ and $4 \geq \sigma(4)$, this implies $\alpha \leq \alpha^{\prime}$. Combining this with the fact that $(\alpha, \beta, \gamma)$ is the biggest monomial in $Q_{2}^{m} Q_{1}^{n}$ with respect to the lexicographic order on $(\alpha, \beta, \gamma)$, one gets $\alpha=\alpha^{\prime}$ and $\sigma(4)=4$. Similarly, $\beta=\beta^{\prime}, \gamma=\gamma^{\prime}$ and $\sigma$ is the identity permutation. This contradiction proves (a), or equivalently

$$
d_{(m, n, 1)}=1 \otimes a^{(\alpha+4)} b^{(\beta+2)} c^{(\gamma+1)}+(\text { other terms }) .
$$

Here and throughout the proof, $\otimes$ means the tensor product over $G L_{3}$.

By definition of the squaring operation

$$
S q^{0}\left(1 \otimes a^{(\alpha+4)} b^{(\beta+2)} c^{(\gamma+1)}\right)=1 \otimes a^{(2 \alpha+9)} b^{(2 \beta+5)} c^{(2 \gamma+3)} .
$$

Now a direct computation using Definition 3.4 shows that

$$
\begin{aligned}
Q_{2} Q_{1} Q_{0} & =x^{12} y^{4} z+x^{10} y^{6} z+x^{10} y^{5} z^{2}+x^{10} y^{4} z^{3} \\
& +x^{9} y^{6} z^{2}+x^{9} y^{5} z^{3}+x^{8} y^{6} z^{3}+x^{8} y^{5} z^{4}+\text { (symmetrized) } .
\end{aligned}
$$

Note that $x^{9} y^{5} z^{3}$ and its symmetrized terms are the only terms of the form $x^{\text {odd }} y^{\text {odd }} z^{\text {odd }}$ in $Q_{2} Q_{1} Q_{0}$. On the other hand,

$$
Q_{2}^{2 m} Q_{1}^{2 n}=x^{2 \alpha} y^{2 \beta} z^{2 \gamma}+\text { (other terms) }
$$

where $x^{2 \alpha} y^{2 \beta} z^{2 \gamma}$ is the biggest monomial in this polynomial with respect to the lexicographic order on $(2 \alpha, 2 \beta, 2 \gamma)$. Focusing on monomials of the form $x^{\text {odd }} y^{\text {odd }} z^{\text {odd }}$ and using the same argument as in the proof of (a), we have

$$
Q_{2}^{2 m+1} Q_{1}^{2 n+1} Q_{0}=x^{2 \alpha+9} y^{2 \beta+5} z^{2 \gamma+3}+\text { (other terms). }
$$

This is equivalent to

$$
1 \otimes a^{(2 \alpha+9)} b^{(2 \beta+5)} c^{(2 \gamma+3)}=d_{(2 m+1,2 n+1,1)}+\text { (other terms). }
$$

Combining (b), (c) and (d), we get

$$
S q^{0}\left(d_{(m, n, 1)}\right)=d_{(2 m+1,2 n+1,1)}+\text { (other terms). }
$$

Applying this for $(m, n, 1)=\left(2^{r}-2^{s}-1,2^{s}-1,1\right)$, we obtain

$$
S q^{0}\left(d_{\left(2^{r}-2^{s}-1,2^{s}-1,1\right)}\right)=d_{\left(2^{r+1}-2^{s+1}-1,2^{s+1}-1,1\right)}+\text { (other terms). }
$$

In addition, $S q^{0}$ maps $P D_{3}^{*}$ to itself (by Proposition 4.2) and $P D_{3}^{*}$ consists of at most one non-zero element of any dimension. So the proposition is proved.

Theorem 5.3 (Hu'ng-Peterson [18]). $P D_{4}^{*}:=P\left(\mathbb{F}_{2} \underset{G L_{4}}{\otimes} H_{*}\left(B V_{4}\right)\right)$ has a basis consisting of

$$
\begin{array}{ll}
d_{\left(2^{s}-1,0,0,0\right)}, & s \geq 0, \\
d_{\left(2^{r}-2^{s}-1,2^{s}-1,1,0\right)}, & r>s>0, \\
d_{\left(2^{t}-2^{r}-1,2^{r}-2^{s}-1,2^{s}-1,2\right)}, & t>r>s>1, \\
d_{\left(2^{r}-2^{s+1}-2^{s}-1,2^{s}-1,2^{s}-1,2\right)}, & r>s+1>2 .
\end{array}
$$

They are of dimensions $2^{s+3}-8,2^{r+3}+2^{s+2}-6,2^{t+3}+2^{r+2}+2^{s+1}-4$ and $2^{r+3}+2^{s+1}-4$, respectively.

Remark. $P D_{4}^{*}$, as well as $P D_{3}^{*}$, has at most one non-zero element of any dimension. 
Proposition 5.4. $S q^{0}: P D_{4}^{*} \rightarrow P D_{4}^{*}$ is given by

$$
\begin{aligned}
& S q^{0}\left(d_{\left(2^{s}-1,0,0,0\right)}\right)=S q^{0}\left(d_{\left(2^{r}-2^{s}-1,2^{s}-1,1,0\right)}\right)=0, \\
& S q^{0}\left(d_{\left(2^{t}-2^{r}-1,2^{r}-2^{s}-1,2^{s}-1,2\right)}\right)=d_{\left(2^{t+1}-2^{r+1}-1,2^{r+1}-2^{s+1}-1,2^{s+1}-1,2\right)}, \\
& S q^{0}\left(d_{\left(2^{r}-2^{s+1}-2^{s}-1,2^{s}-1,2^{s}-1,2\right)}\right)=d_{\left(2^{r+1}-2^{s+2}-2^{s+1}-1,2^{s+1}-1,2^{s+1}-1,2\right)} .
\end{aligned}
$$

Proof. We denote $x_{1}, x_{2}, x_{3}, x_{4}$ by $x, y, z, t$ and $a_{1}, a_{2}, a_{3}, a_{4}$ by $a, b, c, d$, respectively, for brevity.

The first part of the proposition is an immediate consequence of dimensional information.

We claim that $Q_{0}=x^{8} y^{4} z^{2} t+$ (symmetrized). It can be checked by a routine computation using Definition 3.4. Here we give an alternative argument. Indeed, the Dickson algebra $D_{4} \cong \mathbb{F}_{2}\left[Q_{3}, Q_{2}, Q_{1}, Q_{0}\right]$ has exactly one non-zero element of dimension 15. To check the equality we need only to show that the right hand side is $G L_{4}$-invariant. Recall that $G L_{4}$ is generated by the symmetric group $\Sigma_{4}$ and the transformation $x \mapsto x+y, y \mapsto y, z \mapsto z, t \mapsto t$. So, it suffices to check that the right hand side is invariant under this transformation. We leave it to the reader.

Suppose $m, n, p, q$ are non-negative integers with $q>0$. Let $x^{\alpha} y^{\beta} z^{\gamma} t^{\delta}$ be the biggest monomial in $Q_{3}^{m} Q_{2}^{n} Q_{1}^{p} Q_{0}^{q-1}$ with respect to the lexicographic order on $(\alpha, \beta, \gamma, \delta)$. By the same argument as in the proof of Proposition 5.2 we have

$$
Q_{3}^{m} Q_{2}^{n} Q_{1}^{p} Q_{0}^{q}=x^{\alpha+8} y^{\beta+4} z^{\gamma+2} t^{\delta+1}+\text { (other terms). }
$$

In other words,

$$
d_{(m, n, p, q)}=1 \otimes a^{(\alpha+8)} b^{(\beta+4)} c^{(\gamma+2)} d^{(\delta+1)}+(\text { other terms }) .
$$

Here and throughout this proof, $\otimes$ denotes the tensor product over $G L_{4}$.

By definition of the squaring operation

$$
S q^{0}\left(1 \otimes a^{(\alpha+8)} b^{(\beta+4)} c^{(\gamma+2)} t^{(\delta+1)}\right)=1 \otimes a^{(2 \alpha+17)} b^{(2 \beta+9)} c^{(2 \gamma+5)} d^{(2 \delta+3)} .
$$

Using the same method that we used to compute $Q_{0}$ above, we can show that

$$
\begin{aligned}
& Q_{3} Q_{2}=\sum_{\substack{s_{1}+s_{2}+s_{3}+s_{4}=20 \\
s_{i}=0 \text { or a power of } 2}} x^{s_{1}} y^{s_{2}} z^{s_{3}} t^{s_{4}}, \\
& Q_{1}=\sum_{\substack{s_{1}+s_{2}+s_{3}+s_{4}=14 \\
s_{i}=0 \text { or a power of } 2}} x^{s_{1}} y^{s_{2}} z^{s_{3}} t^{s_{4}} .
\end{aligned}
$$

In particular, we have

$$
\begin{aligned}
Q_{3} Q_{2} & =\left(x^{16} y^{2} z t+\text { symmetrized }\right)+(\text { other terms }), \\
Q_{1} & =\left(x^{8} y^{4} z t+\text { symmetrized }\right)+(\text { other terms }) .
\end{aligned}
$$

Here, in both cases, any other term is of the form $x^{\text {even }} y^{\text {even }} z^{\text {even }} t^{\text {even }}$. So

$$
Q_{3} Q_{2} Q_{1}=\left(x^{17} y^{9} z^{5} t^{3}+\text { symmetrized }\right)+(\text { other terms }),
$$

where $x^{17} y^{9} z^{5} t^{3}$ and its symmetrized terms are the only terms of the form $x^{\text {odd }} y^{\text {odd }} z^{\text {odd }} t^{\text {odd }}$ in $Q_{3} Q_{2} Q_{1}$. On the other hand,

$$
Q_{3}^{2 m} Q_{2}^{2 n} Q_{1}^{2 p} Q_{0}^{2 q-2}=x^{2 \alpha} y^{2 \beta} z^{2 \gamma} t^{2 \delta}+\text { (other terms), }
$$

where $x^{2 \alpha} y^{2 \beta} z^{2 \gamma} t^{2 \delta}$ is the biggest monomial in the polynomial with respect to the lexicographic order on $(2 \alpha, 2 \beta, 2 \gamma, 2 \delta)$. Again, we focus on monomials of the form 
$x^{\text {odd }} y^{\text {odd }} z^{\text {odd }} t^{\text {odd }}$ and use the same argument as in the proof of Proposition 5.2 to get

$$
Q_{3}^{2 m+1} Q_{2}^{2 n+1} Q_{1}^{2 p+1} Q_{0}^{2 q-2}=x^{2 \alpha+17} y^{2 \beta+9} z^{2 \gamma+5} t^{2 \delta+3}+\text { (other terms) },
$$

or equivalently

(c)

$1 \otimes a^{(2 \alpha+17)} b^{(2 \beta+9)} c^{(2 \gamma+5)} d^{(2 \delta+3)}=d_{(2 m+1,2 n+1,2 p+1,2 q-2)}+$ (other terms).

Combining (a), (b) and (c), we get

$$
S q^{0}\left(d_{(m, n, p, q)}\right)=d_{(2 m+1,2 n+1,2 p+1,2 q-2)}+\text { (other terms). }
$$

Apply this for $(m, n, p, q)=\left(2^{t}-2^{r}-1,2^{r}-2^{s}-1,2^{s}-1,2\right)$ and $\left(2^{r}-2^{s+1}-2^{s}-\right.$ $\left.1,2^{s}-1,2^{s}-1,2\right)$. Combining the resulting formulas and the facts that $S q^{0}$ maps $P D_{4}^{*}$ to itself (by Proposition 4.2) and that $P D_{4}^{*}$ has at most one non-zero element of any dimension, we obtain the last two formulas of the proposition.

\section{Final Remark}

Recall that $\bar{D}_{k}:=\mathbb{F}_{2} \otimes D_{\mathcal{A}}$. Let

$$
\bar{\Delta}_{D}: \bar{D}_{k} \rightarrow \bigoplus_{\substack{\ell+m=k \\ \ell, m>0}} \bar{D}_{\ell} \otimes \bar{D}_{m}
$$

be the diagonal defined at the beginning of Section 3 .

Conjecture 6.1. (Hu'ng-Peterson [19]). The diagonal $\bar{\Delta}_{D}$ is zero in positive dimensions for any $k>2$.

This conjecture is proved in Lemma 3.3 for $k=3$ and has been proved for $2<k<10$ in [19]. It implies that $j_{k}^{*}$ (respectively, $\varphi_{k}$ ) vanishes on the decomposable elements in $\mathbb{F}_{2} \otimes P H_{*}\left(B V_{k}\right)$ with respect to the product given by Singer [20] and discussed in Section 3 (respectively, in $\operatorname{Ext}_{\mathcal{A}}^{k}\left(\mathbb{F}_{2}, \mathbb{F}_{2}\right)$ with respect to the cup product) for $2<k<10$.

Note added in proof. Conjecture 6.1 has been established by F. Peterson and the author in the final version of [19].

\section{REFERENCES}

1. J. F. Adams, On the non-existence of elements of Hopf invariant one, Ann. Math. 72 (1960), 20-104. MR 25:4530

2. J. F. Adams, Operations of the nth kind in K-theory, and what we don't know about RP ${ }^{\infty}$, New Developments in Topology, G. Segal (ed.), London Math. Soc. Lect. Note Series 11 (1974), 1-9. MR 49:3941

3. M. A. Alghamdi, M. C. Crabb and J. R. Hubbuck, Representations of the homology of BV and the Steenrod algebra I, Adams Memorial Symposium on Algebraic Topology 2, N. Ray and G. Walker (ed.), London Math. Soc. Lect. Note Series 176 (1992), 217-234. MR 94i:55022

4. J. M. Boardman, Modular representations on the homology of powers of real projective space, Algebraic Topology: Oaxtepec 1991, M. C. Tangora (ed.), Contemp. Math. 146 (1993), 49-70. MR 95a:55041

5. W. Browder, The Kervaire invariant of a framed manifold and its generalization, Ann. Math. 90 (1969), 157-186. MR 40:4963

6. D. P. Carlisle and R. M. W. Wood, The boundedness conjecture for the action of the Steenrod algebra on polynomials, Adams Memorial Symposium on Algebraic Topology 2, N. Ray and G. Walker (ed.), Lond. Math. Soc. Lect. Note Series 176 (1992), 203-216. MR 95f:55015 
7. H. Cartan, Puissances divisées, Séminaire H. Cartan, École Norm. Sup., Paris, 1954/55, Exposé 7. MR 19:438c

8. M. C. Crabb and J. R. Hubbuck, Representations of the homology of BV and the Steenrod algebra II, Algebraic Topology: New Trends in Localization and Periodicity (Sant Feliu de Guixois, 1994; C. Broto et al., eds.), Progr. Math. 136, Birkhäuser, 1996, pp. 143-154. CMP 96:15

9. E. B. Curtis, The Dyer-Lashof algebra and the $\Lambda$-algebra, Illinois Jour. Math. 18 (1975), 231-246. MR 51:14054

10. L. E. Dickson, A fundamental system of invariants of the general modular linear group with a solution of the form problem, Trans. Amer. Math. Soc. 12 (1911), 75-98.

11. M. Kameko, Products of projective spaces as Steenrod modules, Thesis, Johns Hopkins University 1990.

12. J. Lannes and S. Zarati, Invariants de Hopf d'ordre supérieur et suite spectrale d'Adams, C. R. Acad. Sci. Paris Sér. I Math. 296 (1983), 695-698. MR 85a:55009

13. J. Lannes and S. Zarati, Sur les foncteurs dérivés de la déstabilisation, Math. Zeit. 194 (1987), 25-59. MR 88j:55014

14. A. Liulevicius, The factorization of cyclic reduced powers by secondary cohomology operations, Mem. Amer. Math. Soc. 42 (1962). MR 31:6226

15. I. Madsen, On the action of the Dyer-Lashof algebra in $H_{*}(G)$, Pacific Jour. Math. 60 (1975), 235-275. MR 52:9228

16. J. P. May, A general algebraic approach to Steenrod operations, Lecture Notes in Math. Vol. 168, Springer-Verlag (1970), 153-231. MR 43:6915

17. N. Minami, On the Hurewicz image of elementary p groups and an iterated transfer analogue of the new doomsday conjecture, Preprint.

18. Nguyễn H. V. Hu'ng and F. P. Peterson, A-generators for the Dickson algebra, Trans. Amer. Math. Soc., 347 (1995), 4687-4728. MR 96c:55022

19. Nguyễn H. V. Hu'ng and F. P. Peterson, Spherical classes and the Dickson algebra, Math. Proc. Camb. Phil. Soc. (to appear).

20. W. Singer, The transfer in homological algebra, Math. Zeit. 202 (1989), 493-523. MR 90i: 55035

21. R. J. Wellington, The unstable Adams spectral sequence of free iterated loop spaces, Memoirs Amer. Math. Soc. 36 (1982), no. 258. MR 83c:55028

22. C. Wilkerson, Classifying spaces, Steenrod operations and algebraic closure, Topology 16 (1977), 227-237. MR 56:1307

Centre de Recerca Matemàtica, Institut d'Estudis Catalans, Apartat 50, E-08193 Bellaterra, Barcelona, España

Current address: Department of Mathematics, University of Hanoi, 90 Nguyễn Trãi Street, Hanoi, Vietnam

E-mail address: nhvhung@it-hu.ac.vn 